\title{
A Statistical Analysis of the Effects of Mistuning on the Forced Response of Cyclic Assemblies ${ }^{1}$
}

\author{
Shih-Tzung Wei ${ }^{2}$, Christophe Pierre ${ }^{4}$ \\ The University of Michigan \\ Ann Arbor, Michigan
}

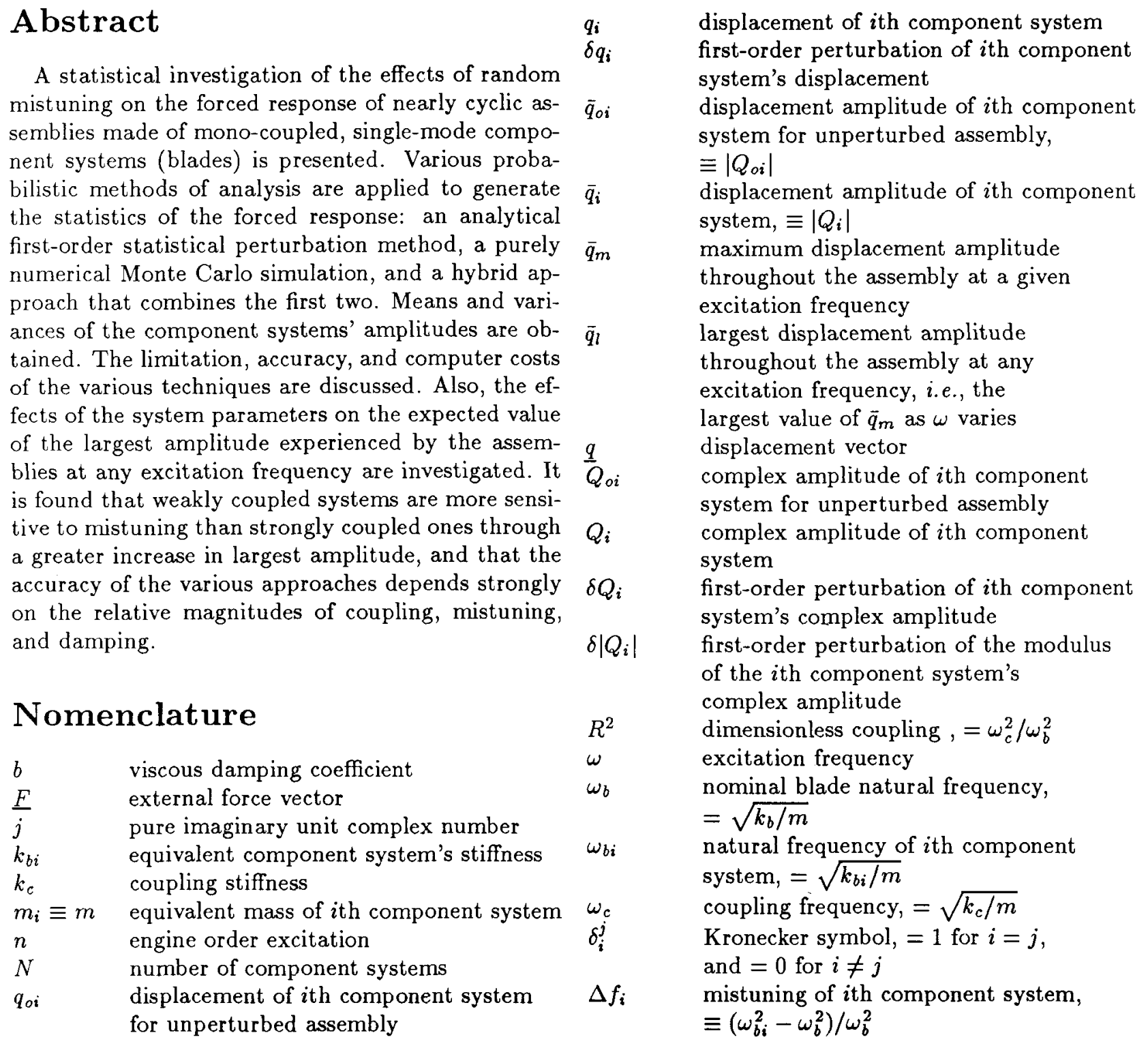

\footnotetext{
${ }^{1}$ Supported in part by NSF Grant MSM-8700820, Dynamic Systems and Control Program.

${ }^{2}$ Graduate Research Assistant.

${ }^{3}$ Member AIAA, ASME. Assistant Professor.

${ }^{4}$ Copyright (C)1989 by Christophe Pierre. Published by the AIAA with permission.
} 


\begin{tabular}{|c|c|}
\hline$\phi_{i}$ & $\begin{array}{l}\text { phase angle of force for } i \text { th componen } \\
\text { system }\end{array}$ \\
\hline$\Delta f$ & standard deviation of mistuning \\
\hline$\sigma_{\left|Q_{i}\right|}$ & $\begin{array}{l}\text { standard deviation of } i \text { th component } \\
\text { system's amplitude }\end{array}$ \\
\hline$\}$ & viscous damping ratio, $=b /\left(2 \sqrt{k_{b} m}\right)$ \\
\hline$T$ & superscript, denotes a transpose \\
\hline$[\bullet]$ & real part of complex number \\
\hline $\mathrm{m}[\bullet]$ & imaginary part of complex number \\
\hline$O(\bullet)$ & order of the argument \\
\hline$E(\bullet)$ & mathematical expectation \\
\hline & modulus \\
\hline & $\equiv \frac{a\left(\frac{1}{d t}\right.}{d t}$ \\
\hline & a \\
\hline
\end{tabular}

\section{Introduction}

Because of manufacturing and material tolerances, some degree of blade mistuning is inevitable in bladed-disk assemblies. Such small mistuning may increase the vibrational amplitudes of some blades significantly and result in the unexpected damage (or failure) of these blades. The extent to which blade mistuning affects the vibrational amplitudes is a major concern in the design of blade assemblies.

A number of studies to predict the increase in the maximum amplitudes experienced by blades have been conducted through the analysis of deterministic mistuning arrangements (see the survey paper by Srinivasan ${ }^{1}$ and the references contained therein). However, the mistuning distribution in a given assembly is randomly selected from a large population of blades. Therefore, the increase in maximum amplitude due to mistuning is expected to vary among the individual ensembles of a population of bladeddisk assemblies. In order to account for the influence of mistuning in the design of bladed-disk assemblies, a statistical analysis of the forced response must be performed by considering the modal properties of the blades as random variables.

Sogliero and Srinivasan ${ }^{2}$ applied Monte Carlo simulation techniques to estimate the fatigue life of mistuned blades. Griffin and Hoosac ${ }^{3}$ also generated the statistics of the forced response by a Monte Carlo simulation of a large number of bladed-disk assemblies. The advantages of Monte Carlo methods lie in the accuracy of the results and the simplicity of the algorithm. However, because of the high computer costs, only limited results can be generated through Monte Carlo simulation, especially for parametric studies of systems with large numbers of degrees of freedom (DOF). Therefore, alternative approaches must be considered to calculate the statistics of the forced response.

Several analytical procedures were developed to generate the statistics of each component system's amplitude. Huang ${ }^{4}$ considered blade mistunings as statistical variables and developed a perturbation method to generate the expectation and variance of the blades' vibrational amplitudes. However, his approach could only be applied to blade assemblies with very closely spaced blades. Sinha ${ }^{5}$ combined first. order perturbation methods with statistical theory to yield the probability density functions of the blades' amplitudes for mistuned systems with an arbitrary number of blades. Although the mistuning distribution was assumed to be Gaussian in this study, Sinha and Chen ${ }^{6}$ later extended the approach to nonGaussian distributions. Nevertheless, the accuracy of these techniques was found to depend strongly upon the amount of damping and blade mistuning. For instance, significant errors were observed for systems with low damping ratios or relatively large mistuning strengths. Also, as will be seen later, some important statistical properties of the forced response such as the mean and variance of the largest amplitude experienced by an assembly cannot be generated through these analytical techniques. Therefore, although such analytical approaches can reduce the computer costs, there are always some significant limitations associated with them. It is very important to identify such limitations before applying those techniques, as otherwise erroneous conclusions could result.

Two issues are addressed in this paper. One is to develop and review various probabilistic approaches to obtain the statistics of the forced response of mistuned systems. The limitations, accuracy, and computer costs of these methods are discussed. The other is to study the effects of the system parameters on the statistics of the forced response of a simple bladeddisk assembly model. Two key parameters governing the sensitivity of the forced response have been shown to be the mistuning strength and the interblade coupling strength. ${ }^{7,8}$ Here, the effects of these parame- 
ters on the expected value of the largest amplitude are studied systematically. This is achieved through the application of Monte Carlo simulation techniques as well as statistical perturbation methods described in section 3 .

\section{Equations of Motion}

The nearly cyclic assembly of $N$ coupled component systems (blades) shown in Fig. 1 is studied. Each component system, consisting of a single-DOF oscillator with viscous damping, is coupled to the adjacent component systems through linear springs. This system has been used by Wei and Pierre ${ }^{7,8}$ as a simple model of continuously shrouded bladed-disk assemblies to study localized free and forced vibrations in mistuned assemblies. For simplicity, mistuning is assumed to originate from discrepancies among the component systems' stiffnesses. The equations of motion are given by

$$
\underline{q}+2 \zeta \omega_{b} \underline{\dot{q}}+\omega_{b}^{2}[A] \underline{q}=\frac{1}{m} \underline{F}
$$

where

$$
[A]=\left[\begin{array}{c}
1+2 R^{2}+\Delta f_{1} \\
-R^{2} \\
0 \\
\vdots \\
0 \\
-R^{2}
\end{array}\right.
$$

$$
\begin{gathered}
-R^{2} \\
+2 R^{2}+ \\
\ddots \\
\ddots \\
\ddots \\
0
\end{gathered}
$$$$
\text { ( }
$$

is a "nearly cyclic" matrix, and

$$
\begin{gathered}
E=F e^{j \omega t}\left\{1, e^{j \phi_{2}}, \cdots, e^{j \phi_{i}}, \cdots, e^{j \phi_{N}}\right\}^{T}, \\
\phi_{i}=\frac{2 \pi n(i-1)}{N}
\end{gathered}
$$

The external force adopted here is a traveling wave excitation which has been widely used for bladeddisk assemblies in the literature. ${ }^{9}$ Two key dimensionless parameters in Eq. (2) are $R^{2}$ and $\Delta f_{i}: R^{2}$ is the dimensionless coupling between component systems and $\Delta f_{i}$ represents the dimensionless mistuning strength for the $i$ th component system. In the subsequent developments $\Delta f_{i}$ is considered a random variable whose statistical properties can be obtained from the survey of a large population of manufactured blades.

\section{Solution Techniques}

The statistics of each blade's vibrational amplitude can be obtained by the following methods.

\subsection{Monte Carlo Simulation}

The Monte Carlo technique is a purely numerical simulation of the probabilistic behavior of the system. It can be outlined by four steps:

1. An ensemble of mistuning values, $\left(\Delta f_{i}\right)_{i=1, \ldots, N}$, is generated from a random variable generator with given statistical distribution;

2. The modal properties of the free undamped system are obtained by solving the $N$-DOF eigenvalue problem numerically;

3. Modal analysis is applied to obtain the force response amplitude of each component system;

$\left.\begin{array}{cc}0 & -R^{2} \\ \cdots & 0 \\ \ddots & \vdots \\ \ddots & 0 \\ \ddots & -R^{2} \\ -R^{2} & 1+2 R^{2}+\Delta f_{N}\end{array}\right]$

4. Steps 1-3 are repeated many (say several hundred) times, thereby generating the statistics of the response amplitudes by averaging over an ensemble of realizations of the mistuned system.

Even though the results are accurate when the sample size is large, Monte Carlo simulations are highly expensive.

\subsection{Hybrid Statistical Perturbation Method Via Free Response}

This method, which combines perturbation theory with Monte Carlo techniques, basically consists of a 
Monte Carlo simulation of the perturbation results. It is different from the Monte Carlo simulation described in the previous paragraph in that the modal properties of the free undamped mistuned system are obtained as perturbations of those of the tuned system (see Ryland II and Meirovitch ${ }^{10}$, Courant and Hilbert ${ }^{11}$, or Appendix A). An advantage of this method is that instead of solving an $N$-DOF eigenvalue problem for each mistuning pattern, only $\left(\frac{N}{2}-1\right)$ if $N$ is even, or $\frac{(N-1)}{2}$ if $N$ is odd, $2-\mathrm{DOF}$ eigenvalue problems need to be solved by this perturbation method (due to the double eigenvalues of the tuned system). Therefore, the computer cost in the second step is significantly reduced. Also, the method retains the accuracy of the Monte Carlo simulations, as second- or even higher-order perturbation schemes can be used.

\subsection{Hybrid Statistical Perturbation Method Via Forced Response}

This method is similar to the one described in the previous paragraph, but here the forced response amplitude of each component system is obtained directly as a perturbation of that of the tuned system (see Appendix B). An advantage of this method is that instead of solving eigenvalue problems and applying modal analysis in steps 2 and 3 , the amplitudes are obtained directly from the perturbation of the forced response of the tuned system. Therefore, the computer cost in the second and third steps is reduced.

\subsection{Analytical Statistical Perturba- tion Method}

Here perturbation methods are combined with multivariate statistical analysis (see Soong and Bogdanoff ${ }^{12}$, Collins and Thomson ${ }^{13}$, and the review paper by Ibrahim ${ }^{14}$ ). From the first-order perturbation method (see Appendix B), the steady-state forced response displacement of each component system can be expressed as

$$
q_{i}=q_{o i}+\delta q_{i} \quad i=1, \ldots, N
$$

where $q_{o i}$ is the displacement of the $i$ th component system for the tuned assembly and $\delta q_{i}$ accounts for the displacement change due to mistuning.
For convenience, the steady-state displacements are written as

$$
\begin{aligned}
q_{i} & =Q_{i} e^{j \omega t} \\
q_{o i} & =Q_{o i} e^{j \omega t} \\
\delta q_{i} & =\delta Q_{i} e^{j \omega t}
\end{aligned}
$$

where $Q_{i}, Q_{o i}$, and $\delta Q_{i}$ are time-independent complex amplitudes which depend on the system parameters and the excitation frequency. $Q_{o i}$ and $\delta Q_{i}$ are obtained in Appendix B. Note that the vibrational amplitude of the $i$ th component system is the modulus of $Q_{i}$. The first-order approximation of the $i$ th component system's amplitude is

$$
\begin{aligned}
\left|Q_{i}\right| & =\left|Q_{o i}+\delta Q_{i}\right| \\
& =\left|Q_{o i}\right|+\delta\left|Q_{i}\right|+\text { H.O.T. }
\end{aligned}
$$

where the first-order variation of the amplitude due to mistuning can easily be shown to be

$$
\delta\left|Q_{i}\right|=\frac{\operatorname{Re}\left(Q_{o i}\right)}{\left|Q_{o i}\right|} \operatorname{Re}\left(\delta Q_{i}\right)+\frac{\operatorname{Im}\left(Q_{o i}\right)}{\left|Q_{o i}\right|} \operatorname{Im}\left(\delta Q_{i}\right),
$$

and the mathematical expectation of the change of the ith component system's amplitude is

$E\left(\delta\left|Q_{i}\right|\right)=\frac{\operatorname{Re}\left(Q_{o i}\right)}{\left|Q_{o i}\right|} \operatorname{Re}\left[E\left(\delta Q_{i}\right)\right]+\frac{\operatorname{Im}\left(Q_{o i}\right)}{\left|Q_{o i}\right|} \operatorname{Im}\left[E\left(\delta Q_{i}\right)\right]$

The expectation (or mean) of $\delta Q_{i}$ is, from Eq. (B.14),

$$
\begin{aligned}
E\left(\delta Q_{i}\right) & =E\left(\sum_{k=1}^{N} \sum_{l=1}^{N} a_{i k l} \Delta f_{l}\right) \\
& =\sum_{k=1}^{N} \sum_{l=1}^{N} a_{i k l} E\left(\Delta f_{l}\right)
\end{aligned}
$$

where $a_{i k l}$ is derived in Appendix B. If the mean value of mistuning is zero, then $E\left(\delta Q_{i}\right)=0$. Therefore, the expectation of each component system's amplitude is

$$
E\left(\left|Q_{i}\right|\right)=\left|Q_{\circ i}\right| \quad i=1, \ldots, N
$$

Hence, to the first order, the mean amplitude of a given component system is the unperturbed one, that is, the tuned system's amplitude. Note, however, that this would not be true for the maximum amplitude throughout the assembly at a given excitation frequency, or for the largest amplitude experienced by 
the assembly at any excitation frequency, for which the mean is not equal to the tuned, or unperturbed, value.

The variance of the $i$ th component system's amplitude is (for mistuning with zero mean)

$$
\begin{aligned}
\sigma_{\left|Q_{i}\right|}^{2}= & E\left[\left(\delta\left|Q_{i}\right|\right)^{2}\right] \\
= & \frac{1}{\left|Q_{o i}\right|^{2}} \sum_{k, l, m, n=1}^{N}\left[\operatorname{Re}\left(Q_{o i}\right) \operatorname{Re}\left(a_{i k l}\right)+\right. \\
& \left.\operatorname{Im}\left(Q_{o i}\right) \operatorname{Im}\left(a_{i k l}\right)\right] \times\left[\operatorname{Re}\left(Q_{o i}\right) \operatorname{Re}\left(a_{i m n}\right)+\right. \\
& \left.\operatorname{Im}\left(Q_{o i}\right) \operatorname{Im}\left(a_{i m n}\right)\right] E\left(\Delta f_{l} \Delta f_{n}\right)
\end{aligned}
$$

where $E\left(\Delta f_{l} \Delta f_{n}\right)$ defines the covariance matrix for the random variables $\left(\Delta f_{i}\right)_{i=1, \ldots, N}$ of mean zero. The $\Delta f_{i}$ 's can be considered to be independent (and therefore uncorrelated) and identically distributed variables, because the blades of a given assembly are selected individually from a large population. Therefore, under this assumption,

$$
E\left(\Delta f_{l} \Delta f_{n}\right)=\sigma_{\Delta f}^{2} \delta_{l}^{n}
$$

where $\sigma_{\Delta f}^{2}$ is the variance of mistuning. Hence, the variance of each component system's amplitude can be simplified as

$$
\begin{aligned}
\sigma_{\left|Q_{i}\right|}^{2}= & \frac{\sigma_{\Delta f}^{2}}{\left|Q_{o i}\right|^{2}} \sum_{k=1}^{N} \sum_{l=1}^{N}\left[\operatorname{Re}\left(Q_{o i}\right) \operatorname{Re}\left(a_{i k l}\right)\right. \\
& \left.+\operatorname{Im}\left(Q_{o i}\right) \operatorname{Im}\left(a_{i k l}\right)\right]^{2}
\end{aligned}
$$

Eqs. (10) and (13) give the expectation and variance of each component system's response amplitude for independent (uncorrelated) blade mistuning variables with zero mean. In general, the variances of the blades' response amplitudes can be obtained easily from Eq. (11) for correlated mistuning variables of given covariance matrix $E\left[\Delta f_{l} \Delta f_{n}\right]$. Also, it is worth noting that the probability density functions of the amplitudes can be derived from Eqs. (7) and (B.14) directly. The advantage of this analytical approach is that it can be applied to systems with any number of blades and to non-Gaussian distributions of the component systems' modal properties.

\section{Results and Discussion}

Here the results obtained by the hybrid and analytical statistical perturbation methods are compared with those of Monte Carlo simulations; the limitations, accuracy and computer costs of the various techniques are discussed. In the Monte Carlo simulations, samples of five hundred assemblies were generated to calculate the statistics of the forced response. Also, the effects of system parameters on the statistics of the forced response are investigated. For simplicity, the statistical distributions of the mistuning, $\Delta f_{i}$, are assumed to be identical and Gaussian with standard deviation $\sigma_{\Delta f}$ and zero mean.

Fig. 2 displays the statistics of the first component system's amplitude, in terms of excitation frequency, obtained by analytical statistical perturbation method (SPM) and by Monte Carlo simulation. The analytical SPM yields very accurate results for this set of parameters and the computer cost was found to be only two hundredths of that of the Monte Carlo simulation. Therefore, the analytical SPM is preferred here to generate the statistics of the individual component system's amplitudes. Unfortunately, not all the statistical properties of the forced response of the assembly can be calculated by the analytical SPM. For instance, the maximum amplitude throughout the assembly at a given excitation frequency can only be obtained by considering the forced response amplitude pattern for each mistuning configuration. Hence, the expected value of the maximum amplitude cannot be derived analytically. In this case, the hybrid SPM must be applied instead.

Fig. 3 shows the expected value of the maximum amplitude in the frequency domain, by both Monte Carlo simulation and hybrid SPM via forced response. The hybrid SPM provides accurate results for this set of parameters without solving the eigenvalue problem and applying modal analysis. However, as mentioned by Sinha ${ }^{5}$ and Wei and Pierre ${ }^{8}$, the accuracy of a perturbation method based on the forced response deteriorates as the ratio of mistuning strength to damping ratio increases. For instance, Fig. 4 is for the same parameters as Fig. 3 except that the damping ratio is decreased to 0.01 . It is observed that the hybrid SPM via forced response significantly overpredicts the Monte Carlo result at excitation frequencies close to the resonant frequency. Therefore, other methods should be considered for such systems with low damping ratios. Here, the hybrid SPM via free response is adopted.

Fig. 5 displays the expected value of the maximum 
amplitude in the frequency domain by Monte Carlo simulation and hybrid SPM via free response. It is shown that for the same set of parameters as in Fig. 4 , the hybrid SPM via free response generates much more accurate results than the hybrid SPM via forced response for this low damping ratio. In fact, the accuracy of the hybrid SPM via free response is insensitive to the value of the damping ratio, which is a clear advantage of the method over a forced response formulation, especially for systems with low damping. Nevertheless, as discussed by Wei and Pierre ${ }^{7}$, the accuracy of this method depends on the ratio of mistuning strength to coupling strength. For weak coupling, very significant errors may be generated by considering mistuning as a perturbation. This is observed in Fig. 6, which compares the hybrid SPM via free response with the Monte Carlo simulation in a weak coupling configuration $(R=0.1)$. In this case, the modified perturbation method described by Wei and Pierre $e^{7}$ that treats the small coupling as the perturbation parameter should be used. However, if the ratio of mistuning to coupling is of the order of one (that is, in the intermediate cases), to the authors' opinion, only Monte Carlo simulations can be applied effectively, as none of the perturbation schemes yield satisfactory results.

An objective of this paper is to carry out a parametric study of mistuning effects. Therefore, in the rest of this section, Monte Carlo simulations are performed to study the effects of the system parameters on the statistics of the forced response. However, results obtained by other approaches are also presented for comparison in some cases.

Fig. 7 shows the expected value of the largest amplitude throughout the assembly at any excitation frequency versus the interblade coupling for various standard deviations of mistuning. It is observed that for the tuned system $\left(\sigma_{\Delta f}=0\right)$ the largest amplitude decreases monotonically as the cuupling increases. Nonetheless, for the mistuned systems, the largest amplitude increases to a maximum value as coupling increases from zero, then decreases as coupling further increases. Also, the increase in largest amplitude due to mistuning is more significant for small values of the interblade coupling. Therefore, weakly coupled systems are more sensitive to mistuning than strongly coupled ones through a greater increase in largest amplitude.
The effect of the number of component systems (blades) on the mean largest amplitude ratio, which is defined as the ratio of the mistuned system's mean largest amplitude to the tuned system's largest amplitude, is shown in Fig. 8. It is observed that, globally, increasing the number of component systems increases the largest amplitude ratio, that is, the effect of mistuning increases as the number of blades increases. Also, it is found that the largest amplitude ratio is larger for weakly coupled systems than for strongly coupled ones; hence, weakly coupled systems are more sensitive to mistuning.

Fig. 9 displays the mean largest amplitude versus the number of component systems for a strongly coupled mistuned system by various statistical methods. Again, one observes that the hybrid SPM via forced response significantly overpredicts the largest amplitudes at this low damping ratio, whereas the hybrid SPM via free response generates very accurate results.

Fig. 10 displays the mean largest amplitude versus the mistuning standard deviation for various coupling values. It is shown that, for any nonzero coupling, the largest amplitude increases linearly as the mistuning standard deviation increases from 0 to approximately 0.1 percent, reaches a maximum value at a certain mistuning standard deviation, then decreases as the mistuning standard deviation further increases. This behavior can be explained by the analytical SPM. It is known from the first-order result of Eq. (13) that the standard deviation of each component system's amplitude increases linearly as the standard deviation of mistuning increases. Therefore, we may expect as well the mean largest amplitude to increase linearly with the mistuning standard deviation. However, the accuracy of the analytical SPM depends on coupling, mistuning, and damping, the method being valid only for $\sigma_{\Delta f} / R^{2} \leq O(1)$ and $\sigma_{\Delta f} / \zeta \leq O(1)$. Hence, for $\zeta=0.001$, the analytical SPM is only valid for mistuning standard deviations approximately smaller than 0.1 percent, which explains that the linear increase of the mean largest amplitude only occurs in the mistuning range from 0 to 0.1 percent. Also note that the slopes of linear increase are different for various interblade couplings and that the slope appears larger for weak coupling, indicating again that weakly coupled systems are more sensitive than strongly coupled ones. From this figure, one can also see that the 
largest amplitude of a mistuned system does not necessarily decrease as the mistuning standard deviation decreases - an interesting result.

Fig. 11 shows the mean largest amplitude versus the mistuning standard deviation for a larger damping ratio $\zeta=0.01$. It is observed that the range of linear increase of the largest amplitude is much greater than that in the previous figure, as it extends to mistuning values of up to 1 percent. Therefore, as the damping ratio increases, one can expect the range of mistuning strength which leads to a linear increase of the largest amplitude to increase. Fig. 12 is for $\zeta=0.1$ and confirms this finding. Finally, it is observed in Figs. 11 and 12 that the hybrid SPM via free response provides very accurate results for the strongly coupled mistuned system; hence, this method can be applied to study the effects of mistuning standard deviation on the forced response of strongly coupled systems efficiently.

\section{Conclusions}

The following conclusions can be drawn from this study:

- Various approaches were presented to calculate the statistics of the forced response of mistuned assemblies. Their accuracy depends on the relative magnitudes of coupling, mistuning, and damping. The analytical SPM and hybrid SPM via forced response are only valid for $\sigma_{\Delta f} / R^{2} \leq O(1)$ and $\sigma_{\Delta f} / \zeta \leq O(1)$. The hybrid SPM via free response can be applied to systems with $\sigma_{\Delta f} / R^{2} \leq O(1)$ and any damping ratio. For systems with $\sigma_{\Delta f} / R^{2}=$ $O(1)$, only Monte Carlo simulation can be used.

- In the design and manufacturing of bladed-disk assemblies, a decrease in mistuning strength (that is, a decrease of the manufacturing tolerances) does not necessarily lead to a decrease in the largest amplitude throughout the assembly. Only in the range $\sigma_{\Delta f} / \zeta \leq$ $O(1)$ is it certain that reductions in manufacturing tolerances decrease the largest amplitude.

- Weakly coupled systems are more sensitive to mistuning than strongly coupled ones through a greater increase in largest amplitude.

\section{References}

[1] Srinivasan, A. V., "Vibrations of Bladed-Disk Assemblies-A Selected Survey," ASME Journal of Vibration, Acoustics, Stress, and Reliability in Design, Vol. 106, 1984, pp. 165-168.

[2] Sogliero, G., and Srinivasan, A. V., "Fatigue Life Estimates of Mistuned Blades via a Stochastic Approach," AIAA Journal, Vol. 18, No. 1, 1980, pp. 318-323.

[3] Griffin, J. H., and Hoosac, T. M., "Model Development and Statistical Investigation of Turbine Blade Mistuning," ASME Journal of Vibration, Acoustics, Stress and Reliability in Design, Vol. 106, 1984, pp. 204-210.

[4] Huang, W., "Vibration of Some Structures With Periodic Random Parameters," AIAA Journal, Vol. 20, No. 7, 1982, pp. 1001-1008.

[5] Sinha, A., "Calculating the Statistics of Forced Response of a Mistuned Bladed Disk Assembly," AIAA Journal, Vol. 24, No. 11, 1986, pp. 17971801.

[6] Sinha, A., and Chen, S., "Probabilistic Analysis of Forced Response of a Bladed Disk Assembly with Various Mistuning Distributions," AIAA Paper 88-2399, Proceedings of the 29th AIAA/ASME/ASCE/AHS Structures, Structural Dynamics and Materials Conference, Williamsburg, Virginia, 1988.

[7] Wei, S.-T., and Pierre, C., "Localization Phenomena in Mistuned Assemblies with Cyclic Symmetry, Part I: Free Vibrations," ASME Journal of Vibration, Acoustics, Stress, and Reliability in Design, Vol. 110, No. 4, 1988, pp. 429-438.

[8] Wei, S.-T., and Pierre, C., "Localization Phenomena in Mistuned Assemblies with Cyclic Symmetry, Part II: Forced Vibrations," ASME Journal of Vibration, Acoustics, Stress, and Reliability in Design, Vol. 110, No. 4, 1988, pp. 439-449.

[9] Griffin, J. H., and Sinha, A., "The Interaction Between Mistuning and Friction in the Forced 
Response of Bladed Disk Assemblies," ASME Journal of Engineering for Gas Turbines and Power, Vol. 107, 1985, pp. 205-211.

[10] Ryland II, G., and Meirovitch, L., "Response of Vibrating Systems with Perturbed Parameters," Journal of Guidance and Control, Vol. 3, 1980, pp. 298-303.

[11] Courant, R., and Hilbert, D., Methods of Mathematical Physics, Vol. 1, Interscience Publishers Inc., New York, 1953, pp. 343-350.

[12] Soong, T. T., and Bogdanoff, J. L., "On the Natural Frequencies of a Disordered Linear Chain of $N$ Degrees of Freedom," Int. J. Mech. Sci., Vol. 5,1963 , pp. 237-265.

[13] Collins, J. D., and Thomson, W. T., "The Eigenvalue Problem for Structural Systems with Statistical Properties," AIAA Journal, Vol. 7, 1969, pp. 642-648.

[14] Ibrahim, R. A., "Structural Dynamics with Parameter Uncertainties," Applied Mechanics Reviews, Vol. 40, No. 3, 1987, pp. 309-328.

\section{Appendix A : Perturbation The- ory for Simple and Multiple Eigenvalues}

Consider the eigenvalue problem

$$
([A]-\lambda[I]) \underline{U}=\underline{0},
$$

where $[A]=\left[A_{o}\right]+[\delta A]$. The eigenvalues and eigenvectors can be approximated by perturbation theory, and one can write, to the first-order:

$$
\begin{gathered}
\underline{U}_{i}=\underline{U}_{o i}+\delta \underline{U}_{i}, \quad i=1, \ldots, N, \\
\lambda_{i}=\lambda_{o i}+\delta \lambda_{i}, \quad i=1, \ldots, N .
\end{gathered}
$$

Assume that only one $\alpha$-fold multiple eigenvalue exists in the unperturbed system, that is, $\lambda_{o 1}=\cdots=$ $\lambda_{o \alpha}=\lambda$, while for $i>\alpha$ the remaining eigenvalues $\lambda_{o i}$ are simple. The first step is to find the suitable set of $\alpha$ eigenvectors associated with $\lambda$ from which to perturb. This suitable set is found from the original eigenvectors $\underline{U}_{o k}$ by means of an orthogonal transformation:

$$
\underline{U}_{o i}^{*}=\sum_{k=1}^{\alpha} a_{i k} \underline{U}_{o k} \quad i=1, \ldots, \alpha
$$

where $a_{i k}$ is determined by solving the following eigenvalue problem,

$$
\sum_{k=1}^{\alpha}\left(D_{k j}-\delta \lambda_{i} \delta_{k}^{j}\right) a_{i k}=0 \quad i, j=1, \ldots, \alpha
$$

where

$$
D_{k j}=\left(\underline{U}_{o j}\right)^{T}[\delta A] \underline{U}_{o k}
$$

and $\delta_{k}^{j}=1$ for $k=j,=0$ otherwise. Assuming that the eigenvalues of (A.5) are distinct, then the orthogonal matrix $\left[a_{i k}\right]$ and the $\delta \lambda_{i}$ 's are determined uniquely. Let us select our new unperturbed eigenvectors as $\underline{U}_{o i}^{*}$ given by Eq. (A.4) for $i=1, \ldots, \alpha$, and $\underline{U}_{o i}^{*}=\underline{U}_{o i}$ for $i>\alpha$, and choose their norms to be 1. Also define $d_{j i}=\left(\underline{U}_{o i}^{*}\right)^{T}[\delta A] \underline{U}_{o j}^{*}$. The first-order perturbation of the eigenvalue is given by

$$
\delta \lambda_{i}=d_{i i} \quad i=1, \ldots, N
$$

and the first-order perturbation of the eigenvector is,

$$
\delta \underline{U}_{i}=\sum_{j=1}^{N} \varepsilon_{i j} \underline{U}_{o j}^{*} \quad i=1, \ldots, N
$$

where

$$
\varepsilon_{i i}=0
$$

and

$$
\begin{gathered}
\varepsilon_{i j}=\frac{d_{i j}}{\lambda_{o i}-\lambda_{o j}} \quad i>\alpha \text { or } j>\alpha, \quad i \neq j \\
\varepsilon_{i j}=\frac{1}{d_{i i}-d_{j j}} \sum_{k=\alpha+1}^{N} \frac{d_{i k} d_{k j}}{\lambda_{o i}-\lambda_{o k}} \\
i \leq \alpha \text { and } j \leq \alpha, \quad i \neq j
\end{gathered}
$$

The case where all eigenvalues are simple is a special case of the above results for $\alpha=1$.

For the system studied in this paper, the unperturbed matrix, $\left[A_{0}\right]$, has $\frac{N-1}{2}$ (resp. $\frac{N}{2}$ ) double eigenvalues for $N$ odd (resp. even). Therefore, Eqs. (A.4A.6) must be repeatedly solved $\frac{N-1}{2}\left(\right.$ resp. $\left.\frac{N}{2}\right)$ times 
for $N$ odd (resp. even) with $\alpha=2$ to obtain the suitable set of eigenvectors from which to perturb. Then, Eqs. (A.7-A.11) are applied to obtain the first-order perturbation of eigenvalues and eigenvectors.

\section{Appendix B: Perturbation Method Via Forced Response of Tuned System}

The forced response of the strongly coupled mistuned system is obtained as a perturbation of that of the corresponding tuned system. From the equation of motion

$$
\underline{\ddot{q}}+2 \zeta \omega_{b} \underline{\dot{q}}+\omega_{b}^{2}[A]\{q\}=\frac{1}{m} \underline{F}
$$

let

$$
\begin{aligned}
& \underline{q}=\underline{q_{o}}+\delta \underline{q} \\
& {[A]=\left[A_{o}^{c}\right]+\left[\delta A^{c}\right]} \\
& {\left[\delta A_{c}\right]=\left[\begin{array}{ccccc}
\Delta f_{1} & 0 & \cdots & \cdots & 0 \\
0 & \ddots & \ddots & \ddots & \vdots \\
\vdots & \ddots & \Delta f_{i} & \ddots & \vdots \\
\vdots & \ddots & \ddots & \ddots & 0 \\
0 & \cdots & \cdots & 0 & \Delta f_{N}
\end{array}\right]}
\end{aligned}
$$$$
\left[\begin{array}{cccccc}
1+2 R^{2} & -R^{2} & 0 & \cdots & 0 & -R^{2} \\
-R^{2} & 1+2 R^{2} & -R^{2} & 0 & \cdots & 0 \\
0 & \ddots & \ddots & \ddots & \ddots & \vdots
\end{array}\right]
$$$$
\left[A_{o}^{c}\right]=\left[\begin{array}{cccccc}
0 & \ddots & \ddots & \ddots & \ddots & \vdots \\
\vdots & \ddots & \ddots & \ddots & \ddots & 0 \\
0 & \ddots & \ddots & \ddots & \ddots & -R^{2} \\
-R^{2} & 0 & \cdots & 0 & -R^{2} & 1+2 R^{2}
\end{array}\right]
$$

(B4)

$q_{o}$ is the forced response of the tuned system, and $\delta \underline{q}$ accounts for the effect of mistuning.

Introducing Eqs. (B.2, B.3) into Eq. (B.1) and equating the zeroth- and first-order terms, one obtains

$$
\begin{gathered}
\ddot{q}_{o}+2 \zeta \omega_{b} \underline{\dot{q}_{o}}+\omega_{b}^{2}\left[A_{o}^{c}\right] \underline{q_{o}}=\frac{1}{m} \underline{F} \\
\delta \underline{\ddot{q}}+2 \zeta \omega_{b} \delta \underline{\dot{q}}+\omega_{b}^{2}\left[A_{o}^{c}\right] \delta \underline{q}=-\omega_{b}^{2}\left[\delta A^{c}\right] \underline{q_{o}}
\end{gathered}
$$

The solution of Eq. (B.6) is the forced response of the tuned system and can be obtained by modal analysis. The displacement of each component system is

$$
q_{o i}=Q_{o i} e^{j \omega t} \quad i=1, \ldots, N
$$

where

$$
Q_{o i}=\left(\frac{F e^{j \phi_{i}}}{m \omega_{b}^{2}}\right) \frac{1}{\left(\lambda_{n+1}-\frac{\omega^{2}}{\omega_{b}^{2}}\right)+j\left(2 \zeta \frac{\omega}{\omega_{b}}\right)}
$$

and

$$
\lambda_{n+1}=1+2 R^{2}\left(1-\cos \frac{2 \pi n}{N}\right) .
$$

Eq. (B.7) can be decoupled by using the same modal matrix, $[P]$, whose columns are the eigenvectors of the tuned system, $\underline{U}_{o i}$. Introducing $\delta \underline{q}=[P] \delta \underline{\eta}$ into Eq. (B.7), $\delta \underline{\eta}$ is obtained as

$$
\delta \eta_{i}=-\frac{T_{i}}{M_{i}} \underline{U}_{o i}^{T}\left[\delta A^{c}\right] \underline{q_{o}} \quad i=1, \ldots, N
$$

where

$$
T_{i}=\frac{1}{\left(\lambda_{o i}-\frac{\omega^{2}}{\omega_{b}^{2}}\right)+j\left(2 \zeta \frac{\omega}{\omega_{b}}\right)} \quad i=1, \ldots, N
$$

and $M_{i}$ is the generalized mass (the square of the norm of $\underline{U}_{o i}$ ). The first-order perturbation of each component system's displacement is obtained as

$$
\delta q_{i}=\sum_{k=1}^{N} P_{i k} \delta \eta_{k}=\delta Q_{i} e^{j \omega t} \quad i=1, \ldots, N
$$

where

$$
\begin{gathered}
\delta Q_{i}=\sum_{k=1}^{N} \sum_{l=1}^{N} a_{i k l} \Delta f_{l} \\
a_{i k l}=-\frac{T_{k}}{M_{k}} P_{i k} P_{l k} Q_{o i}
\end{gathered}
$$

where $P_{i k}$ and $P_{k l}$ are elements of the modal matrix $[P]$. 


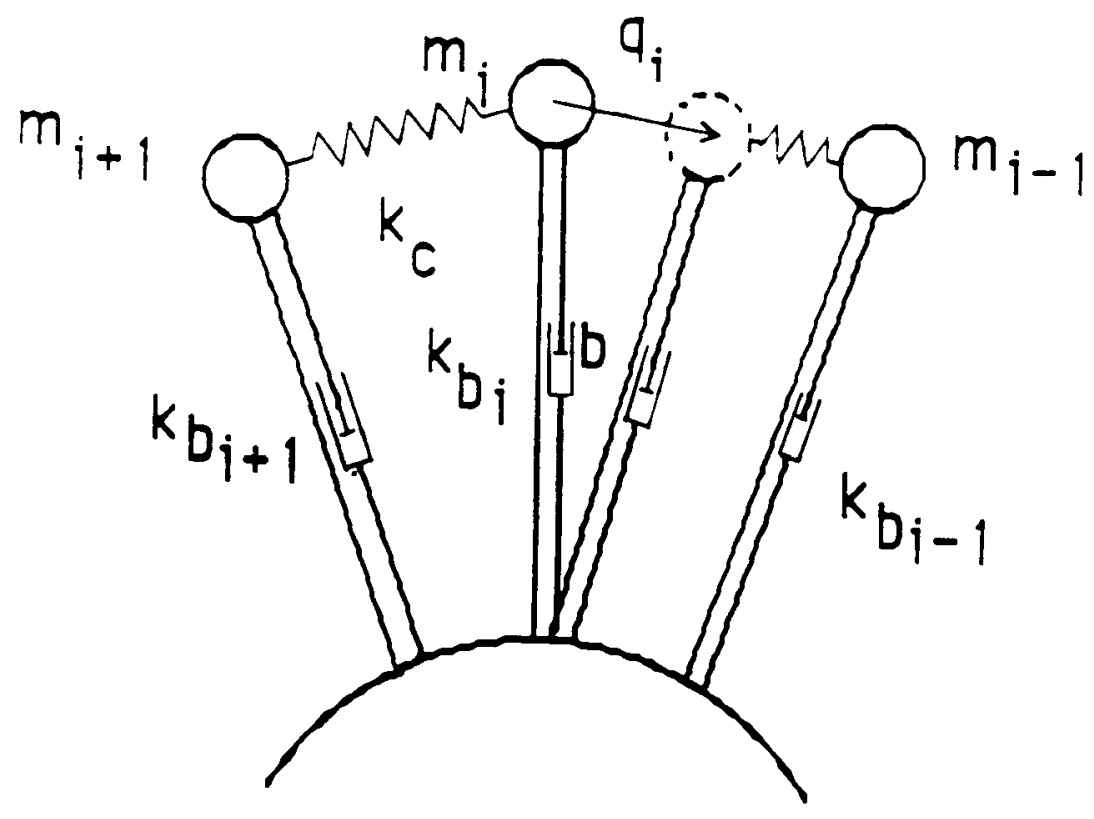

Figure 1: Geometry of nearly periodic structure with cyclic symmetry.

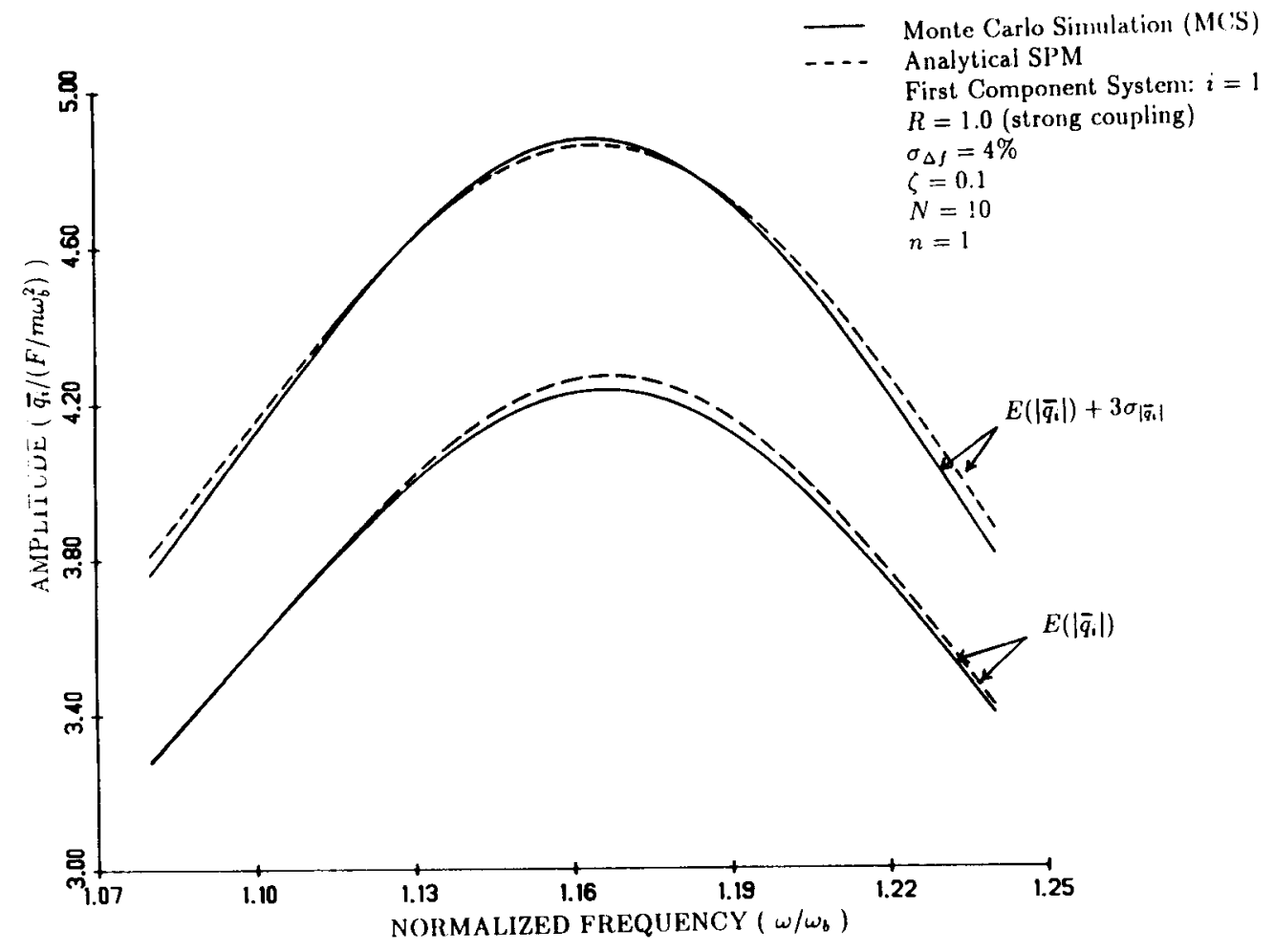

Figure 2: Comparison of the expected value and standard deviation (upper three- $\sigma$ bound) of the first component system's frequency response by analytical SPM and Monte Carlo simulation, for $\zeta=0.1$ and $R=1.0$. 


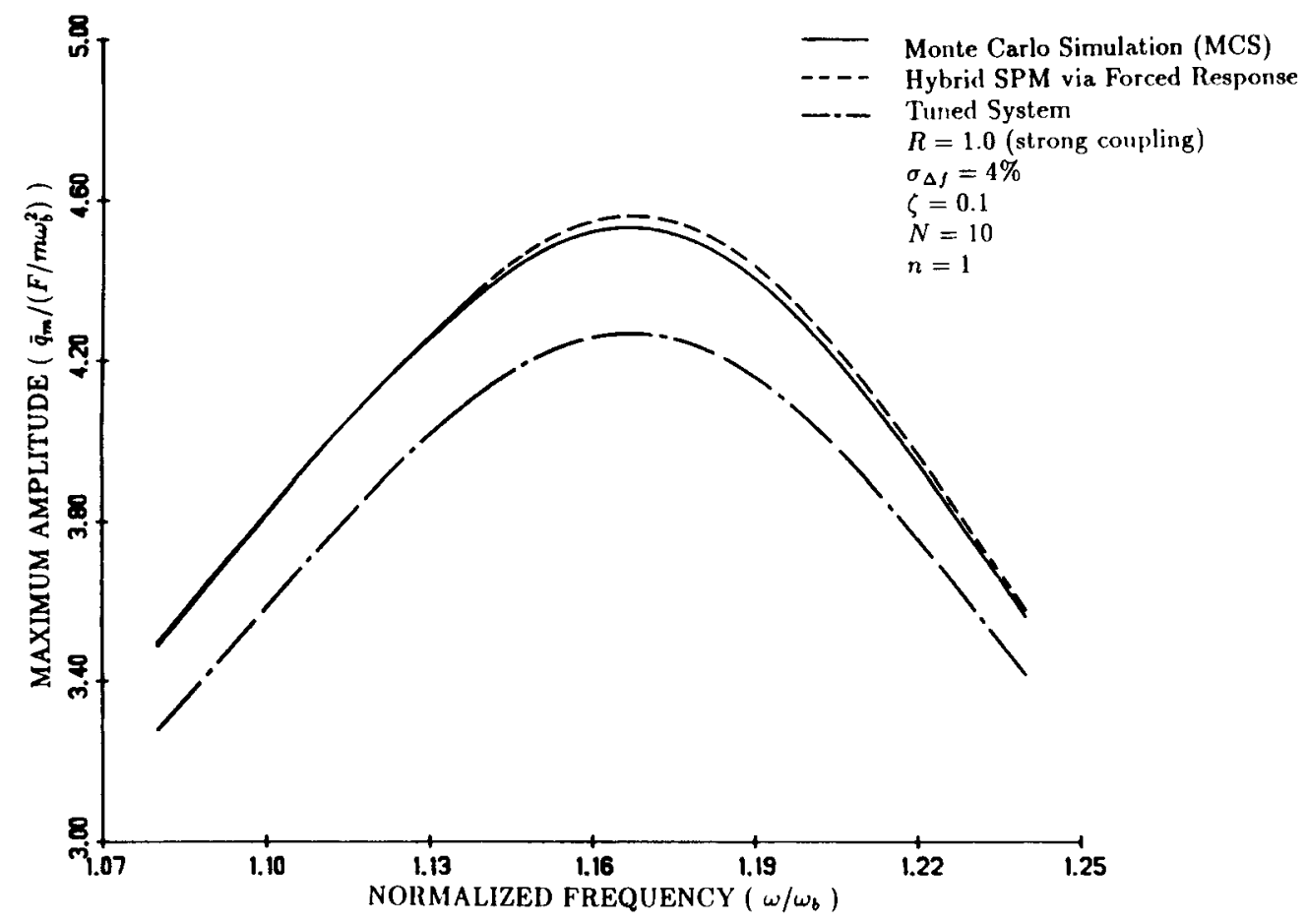

Figure 3: Comparison of the mean maximum amplitude by hybrid SPM via forced response and by Monte Carlo simulation, for $\zeta=0.1$ and $R=1.0$.

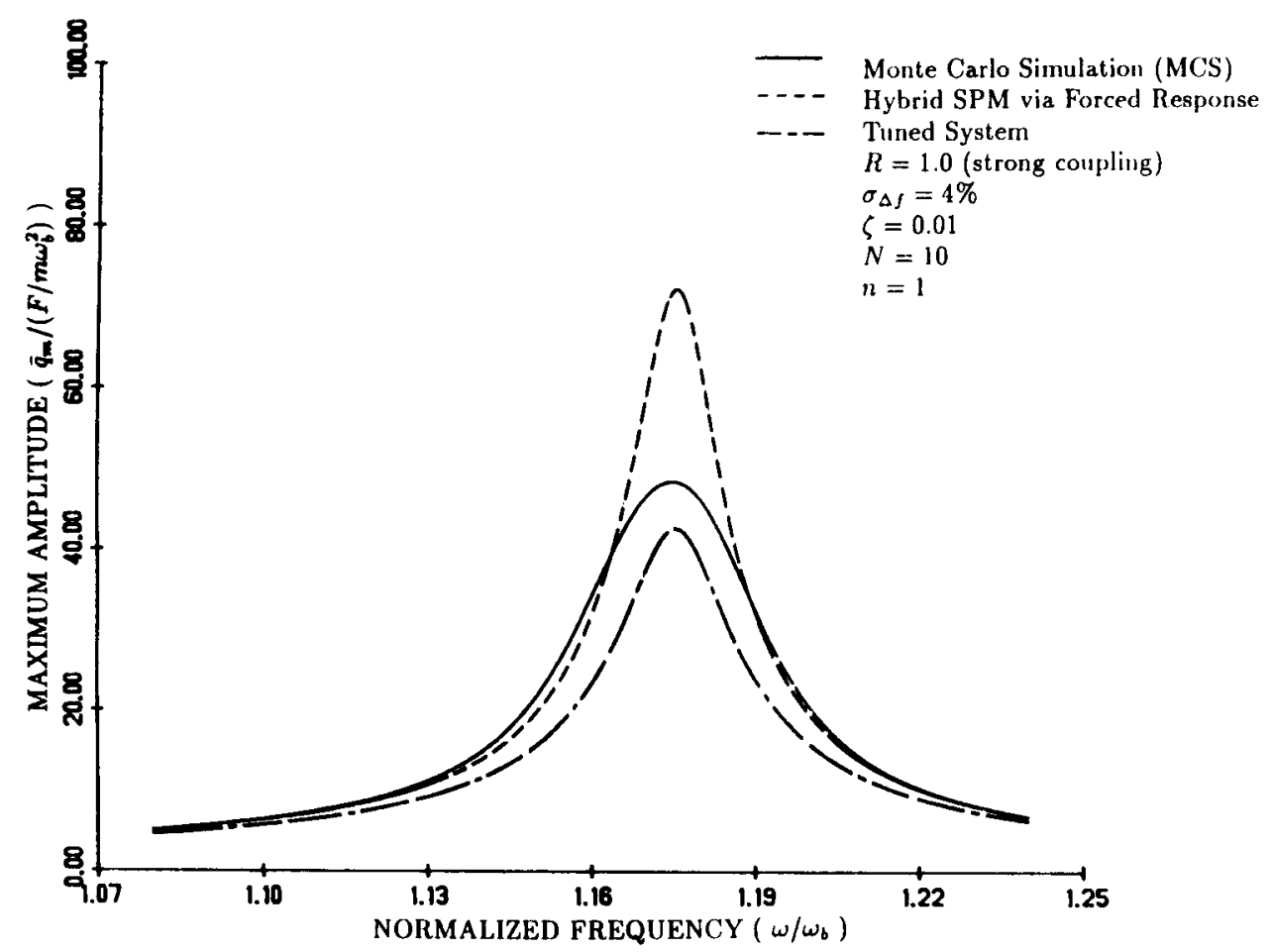

Figure 4: Comparison of the mean maximum amplitude by hybrid SPM via forced response and by Monte Carlo simulation, for $\zeta=0.01$ and $R=1.0$. 


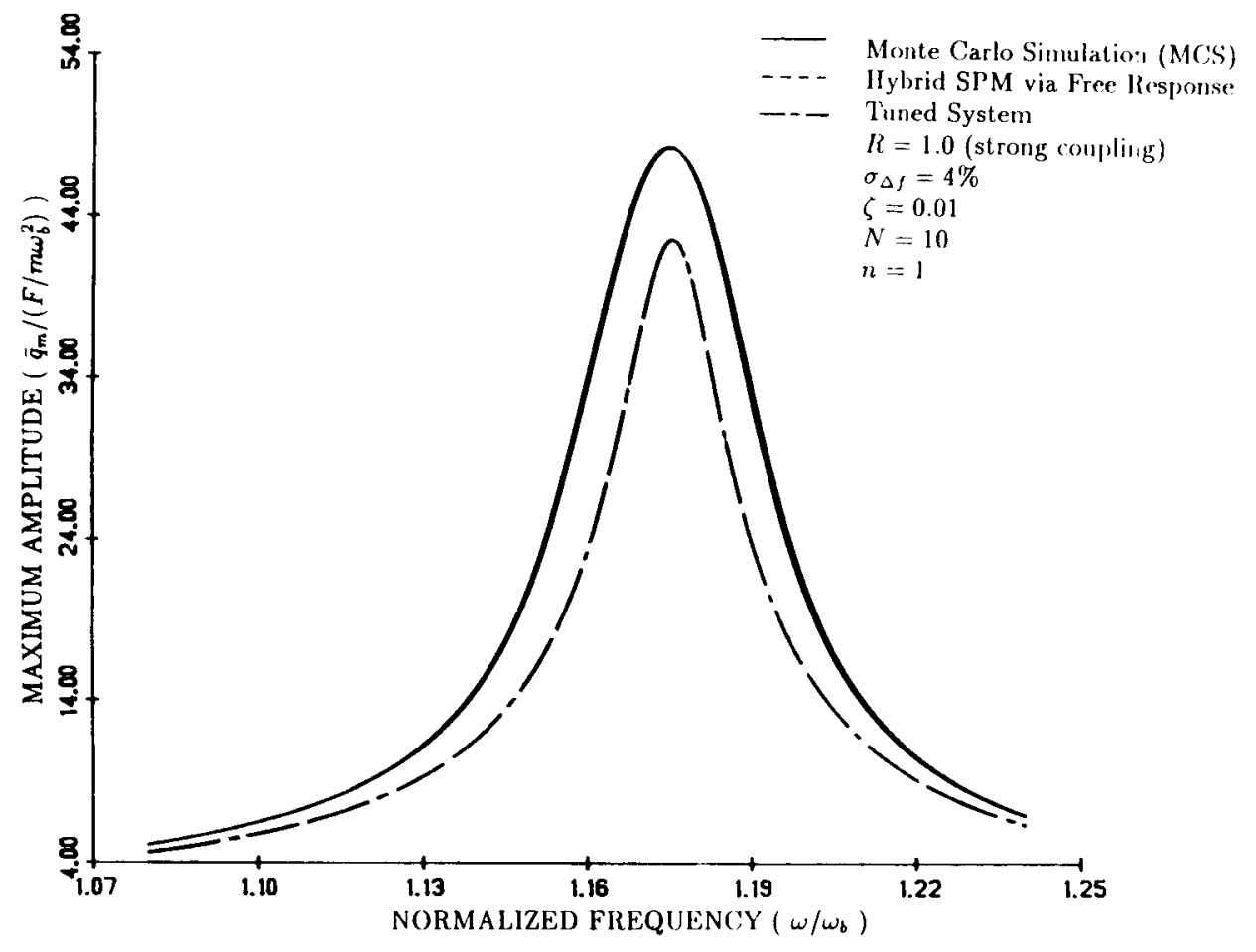

Figure 5: Comparison of the mean maximum amplitude by hybrid SPM via free response and by Monte Carlo simulation, for $\zeta=0.01$ and $R=1.0$.

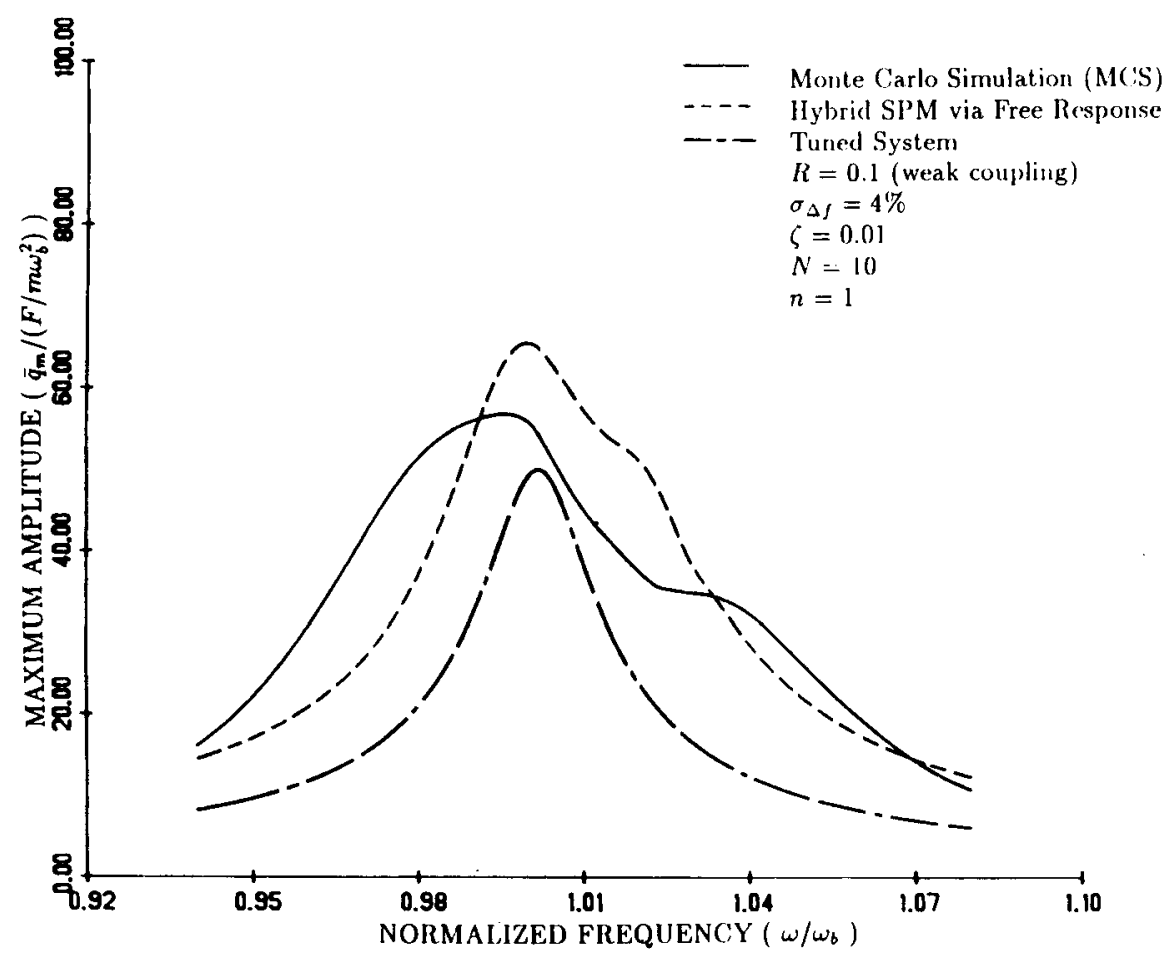

Figure 6: Comparison of the mean maximum amplitude by hybrid SPM via free response and by Monte Carlo simulation, for $\zeta=0.01$ and $R=0.1$. 


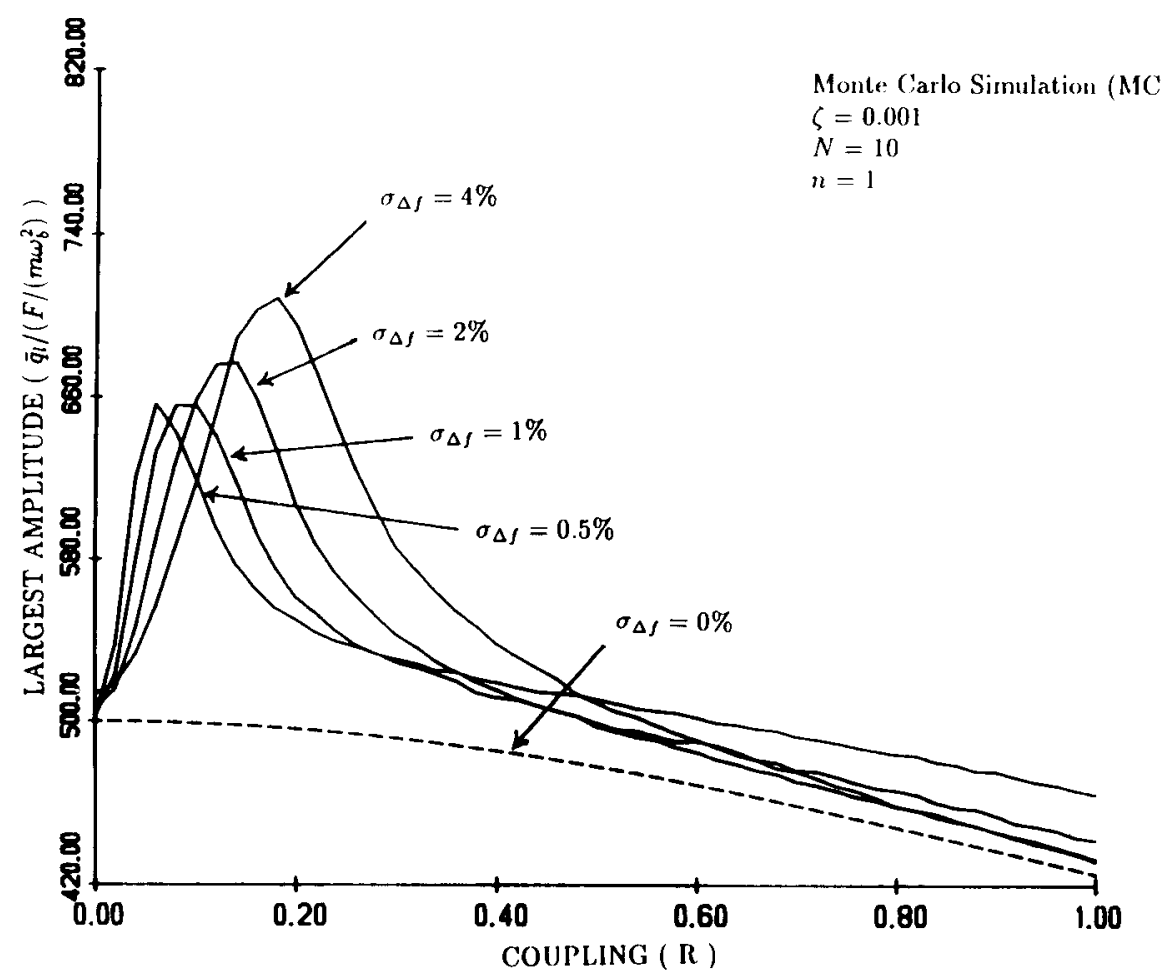

Figure 7: Mean largest amplitude throughout the assembly at any excitation frequency versus coupling, for $\zeta=0.001$ and various mistuning standard deviations.

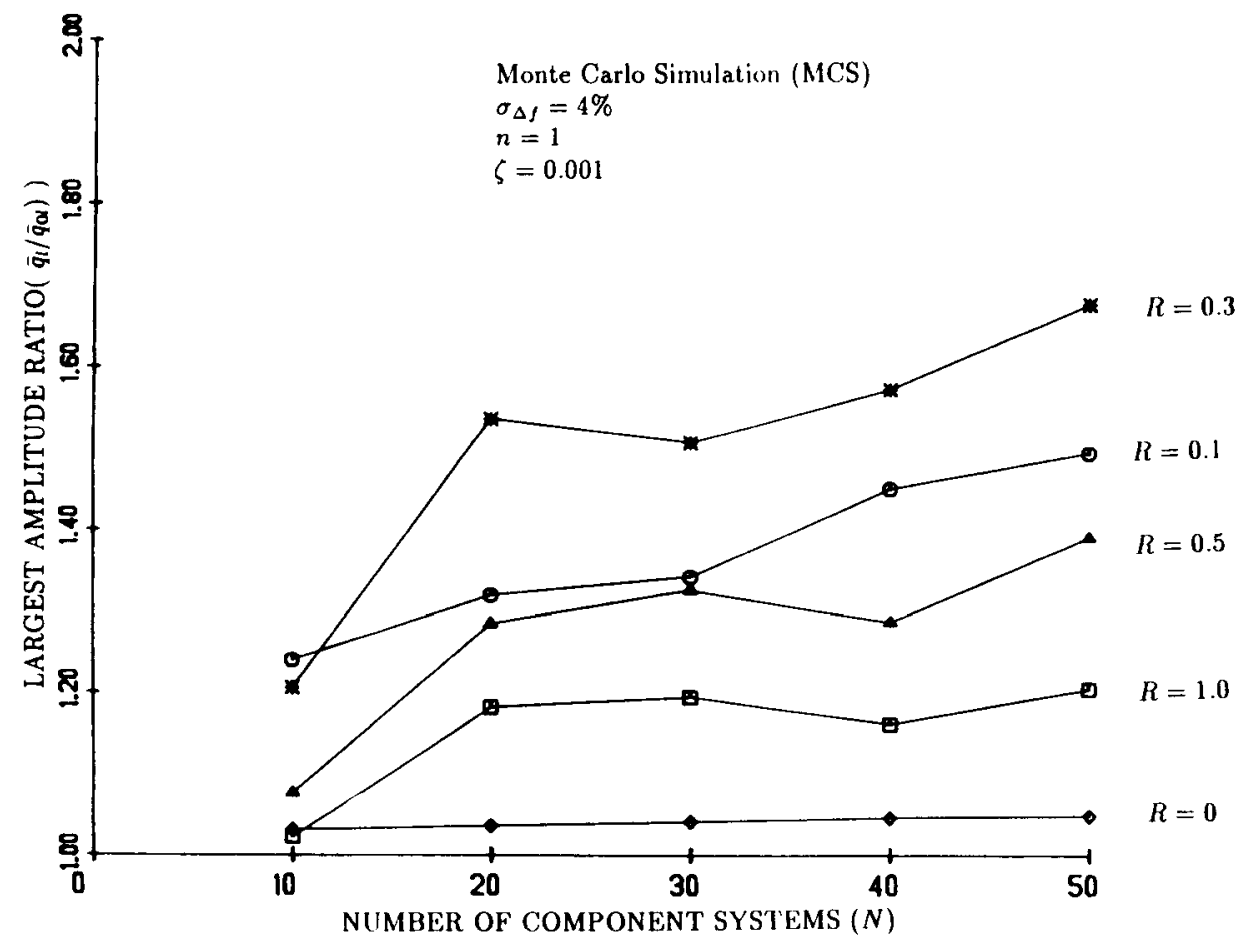

Figure 8: Mean largest amplitude ratio throughout the assembly at any excitation frequency versus number of component systems, for $\zeta=0.001$ and various couplings. 


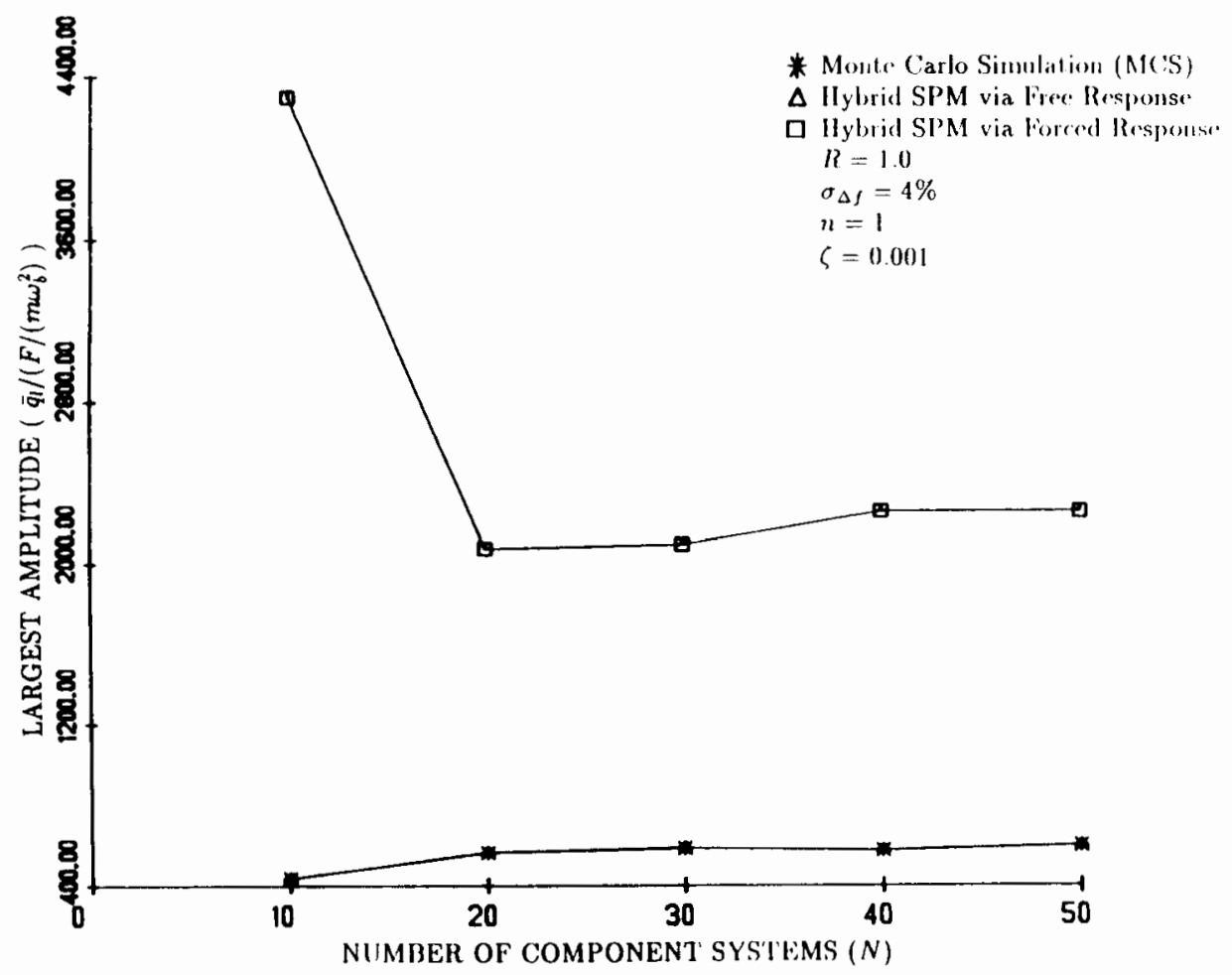

Figure 9: Comparison of the mean largest amplitude by hybrid SPM via forced response, hybrid SPM via free response, and Monte Carlo simulation, for $\zeta=0.001, R=1.0$, and various numbers of component systems.

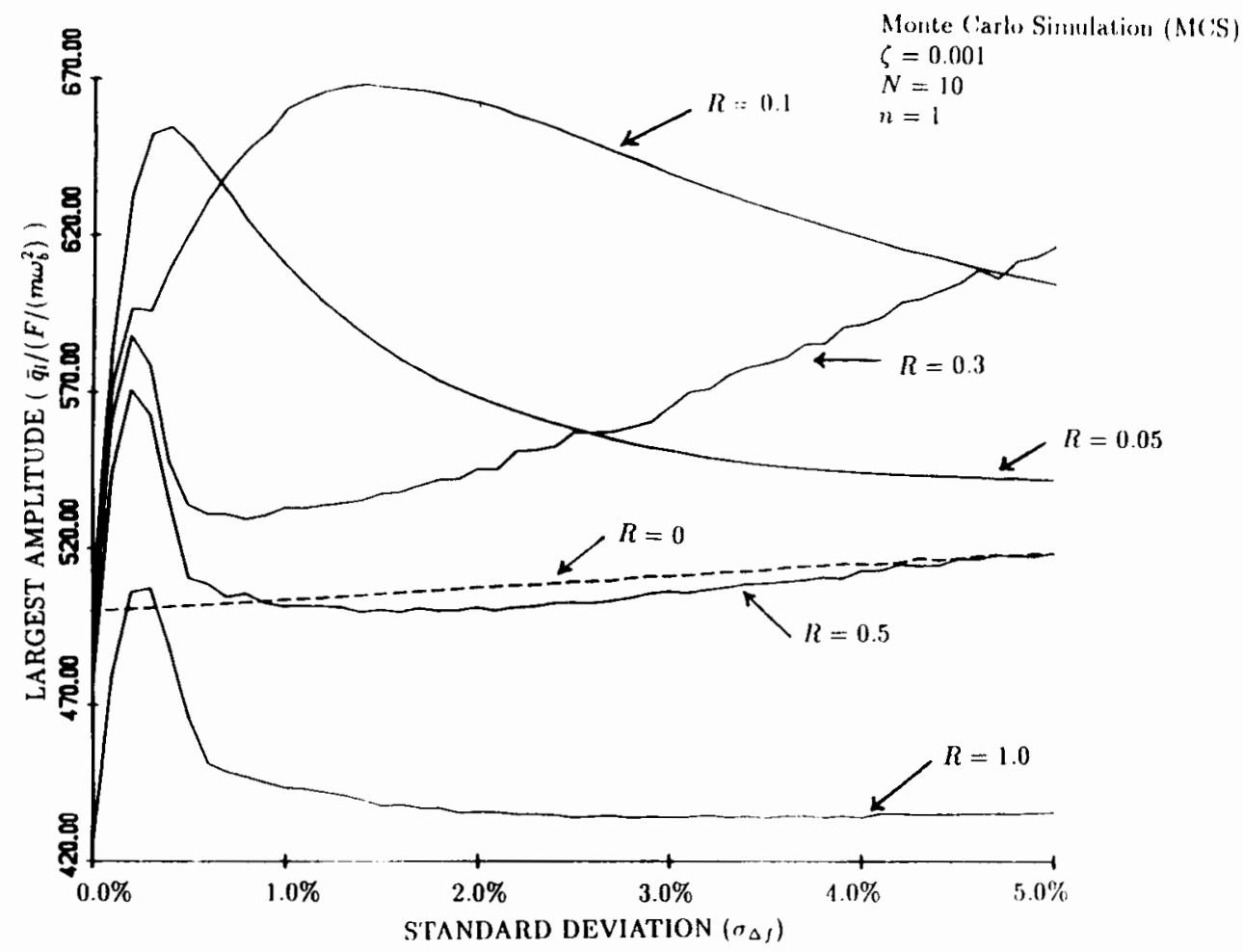

Figure 10: Mean largest amplitude throughout the assembly at any excitation frequency versus mistuning standard deviation, for $\zeta=0.001$ and various couplings. 


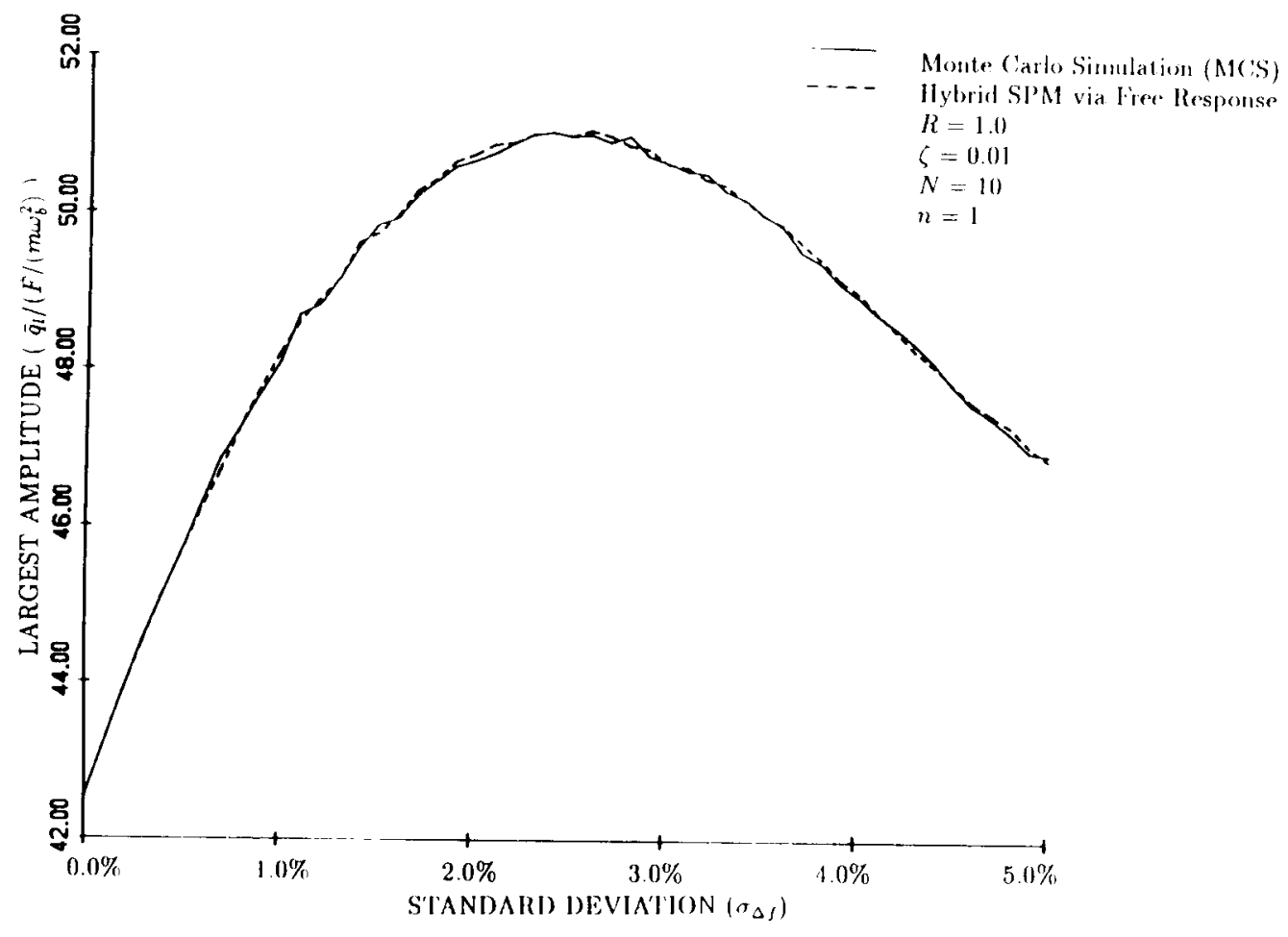

Figure 11: Mean largest amplitude throughout the assembly at any excitation frequency versus mistuning standard deviation, for $\zeta=0.01$ and $R=1.0$.

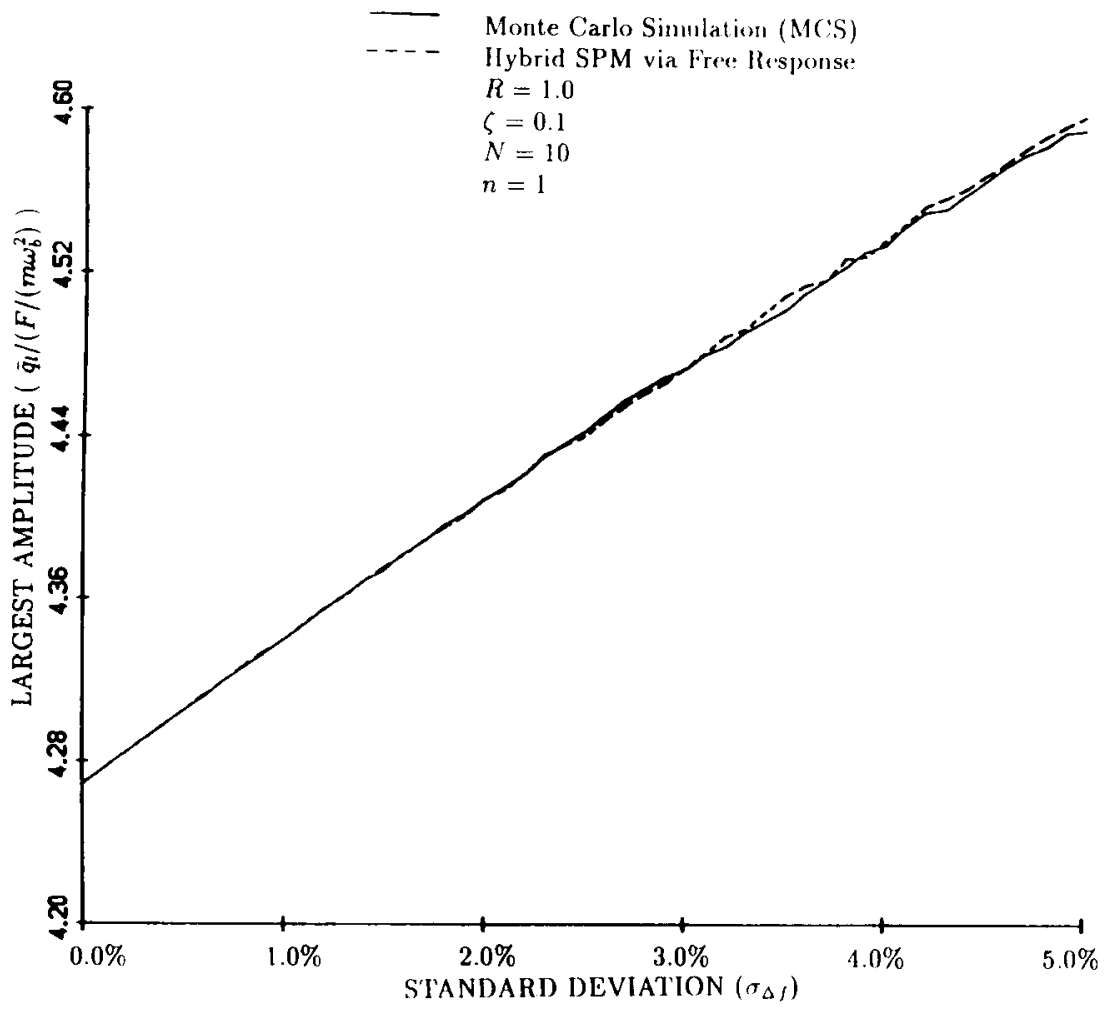

Figure 12: Mean largest amplitude throughout the assembly at any excitation frequency versus mistuning standard deviation, for $\zeta=0.1$ and $R=1.0$. 